\title{
Approche quantitative et géochimique du gradient altitudinal des précipitations sur un bassin versant partiellement englacé sur la côte occidentale du Spitsberg
}

\author{
Quantitative and geochemical approach of altitude precipitation \\ on a catchment area partially covered by a glacier on the occidental coast \\ of Spitsberg
}

par Madeleine Griselin, Laboratoire Environnement Paysage, Théma, CNRS, Besançon

et Christelle Marlin,

Laboratoire d'Hydrologie et de Géochimie isotopique, Université Paris-Sud, Orsay

In order to study water circulation of a small catchment, partially covered by a glacier in the Brogger peninsula area (Svalbard), quantitative and isotopic approaches give information on the recharge water of the hydrological and glaciological systems e.g. the meteoric water. The altitude effect has been identified by different parameters as the snow amount and the isotopic composition in ${ }^{18} \mathrm{O}$ and ${ }^{2} \mathrm{H}$. The area at 400 m.a.s.l seems to receive more precipitation than the other altitudes of the catchment. If the snowfall appears to be more depleated in heavy isotopes in altitude, the effect of both strong winds and morphology of the catchment can induce a disruption of the initial geochemical signal.

\section{INTRODUCTION}

Depuis de nombreuses années, des études hydro-glaciologiques et géochimiques sont réalisées sur le bassin du glacier Loven Est, côte nord-ouest du Spitsberg (entre autres [1, 2, 3]. Définir la recharge de ce bassin versant, englacé à $60 \%$, de petite dimension $\left(10 \mathrm{~km}^{2}\right)$ et possédant un relief contrasté (0 à $876 \mathrm{~m})$, a nécessité d'évaluer le double impact de l'altitude sur les précipitations - principale fonction " entrée " des systèmes hydrologiques -, à la fois sur les quantités précipitées mais aussi sur leur composition isotopique en ${ }^{18} \mathrm{O}$ et ${ }^{2} \mathrm{H}$. La faible surface du bassin versant étudié laisse à penser qu'a priori la variabilité de la fonction " entrée " est faible et que la définition de cette dernière en un nombre limité de points de mesure pourrait suffire. Les mesures de teneurs en oxygène18 et deutérium ont été réalisées sur les eaux de pluie et de neige prélevées afin :

- d'évaluer l'effet de la variation d'altitude sur la signature isotopique des précipitations ;

- de pouvoir comparer la signature isotopique des précipitations à celle des eaux sous-glaciaires et des eaux circulant dans la moraine frontale ;
- d'identifier les zones d'alimentation en eau du réseau intra- et sous-glaciaire.

Dans cet article, seule l'approche quantitative et géochimique du gradient altitudinal des précipitations sera abordée.

\section{II $\square$ MÉTHODOLOGIE}

Proche de la base française de la baie du Roi, le Loven Est est tout à fait représentatif des petits glaciers de type alpin du Spistberg, en pleine phase de retrait derrière un appareil morainique surdimensionné, correspondant au maximum du petit âge glaciaire (Fig. 1). Le vallum morainique bute contre une barre calcaire, d'âge permo-carbonifère, véritable barrière aux écoulements, dans laquelle les torrents issus du glacier ont forcé deux passages en canyons.

Sur le plan climatique, le Loven Est appartient aux marges polaires, il est soumis à l'extrême variabilité du climat polaire océanique (moyennes annuelles à $\mathrm{Ny}$ Aalesund : $\mathrm{T}=-5,9^{\circ} \mathrm{C}$ et $\left.\mathrm{P}=392 \mathrm{~mm},[4]\right)$.

Etudié depuis plus de trente ans, le Loven Est est l'objet d'études fines mais ponctuelles dans le temps : les durées relativement courtes des observations ne permettent pas 


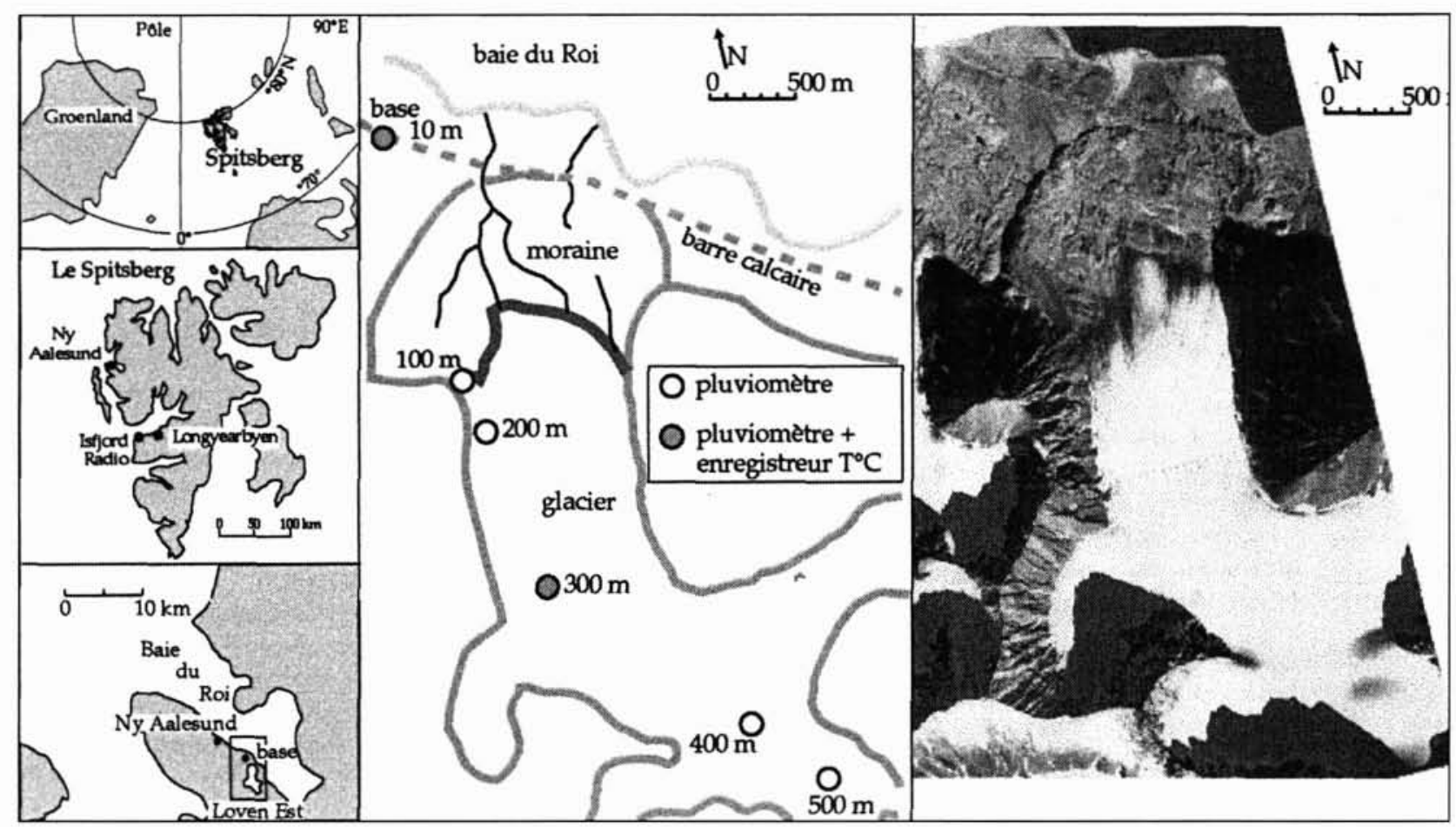

1. Le bassin du Loven Est, $79^{\circ}$ Nord, Spitsberg : implantation des stations de mesure des températures et des précipitations.

d'avoir des séries très longues. En revanche, les chroniques météorologiques de Ny Aalesund (station Norske Meteorologiske Institutt) ont plus de trente ans et concernent un lieu situé à $6 \mathrm{~km}$ à l'est de la base française, à $10 \mathrm{~m}$ d'altitude, en même rive du fjord. Les corrélations établies entre les paramètres climatiques de $\mathrm{Ny}$ Aalesund et la base française ont montré un gradient de continentalité négligeable qui autorise l'utilisation des valeurs de $\mathrm{Ny}$ Aalesund pour la partie avale du bassin versant du Loven Est [5].

En complément de mesures réalisées en 1993 et 1995 sur différentes colonnes de neiges sur le bassin versant, l'un des volets de la campagne d'automne 1998 (01.09 au 10.10) était l'établissement de gradients altitudinaux en ce qui concerne les précipitations. A cette époque de l'année, les précipitations sont presque toujours neigeuses au niveau de la mer. Le temps est relativement instable et les vents, souvent violents, soufflent presque systématiquement après chaque chute de neige, ce qui rend difficile les mesures et la collecte d'échantillons de précipitations. Six pluviomètres couplés à des thermomètres maxi-mini ont été installés tous les $100 \mathrm{~m}$ d'altitude, de 10 à $520 \mathrm{~m}$ (enregistreur de la température de l'air et son humidité relative à 10 et $300 \mathrm{~m}$ ). Ces stations consistaient en un trépied, solidement ancré à la glace ou au sol, auquel étaient suspendus les pluviomètres. Après chaque événement majeur de précipitation, les pluviomètres étaient vidés dans le but de recueillir l'intégralité de la neige pour mesurer la lame d'eau tombée depuis le prélèvement précédent et récolter des échantillons pour analyses isotopiques. En laboratoire, les teneurs isotopiques en oxygène 18 et en deutérium ont été déterminées suivant les protocoles expérimentaux de Mc Crea [6] et Coleman et al. [7]. L'incertitude sur ces mesures est de $0,2 \%$ pour l'oxygène 18 et de $2 \%$ pour le deutérium.

\section{III — RÉSULTATS ET INTERPRÉTATION}

\subsection{Episodes de précipitations observés}

En complément des mesures effectuées sur le Loven Est, les données climatiques enregistrées à $\mathrm{Ny}$ Aalesund ont été utilisées : hauteur de précipitations, température instantanée, force et direction des vents. Les températures instantanées, mesurées quatre fois par jour à $\mathrm{Ny}$ Aalesund, sont représentatives de celles de l'aval du bassin Loven-Est (Fig. 2). Un écart quasi constant, de 3 à $5^{\circ} \mathrm{C}$, a été par ailleurs observé entre les enregistrements de températures à 10 et à $300 \mathrm{~m}$ d'altitude (soit un gradient de 1,0 à $1,7^{\circ} \mathrm{C}$ par $100 \mathrm{~m}$ pour cette période).

Cinq épisodes majeurs de précipitation ont eu lieu pendant la période d'observation (Fig. 2) :

- le 4 septembre (pluie à $10 \mathrm{~m}$, neige dès $100 \mathrm{~m}$ ) ;

- les 7, 28 septembre, le $1^{\text {er }}$ et 7 octobre (neige à toutes les altitudes).

Les prélèvements correspondants $(5,8$ et 29 septembre, 8 octobre) sont portés sur la figure 2 avec une hauteur proportionnelle à la lame d'eau précipitée à $100 \mathrm{~m}$ d'altitude et une largeur montrant la période couverte entre deux prélèvements. Les derniers épisodes neigeux sont de loin les plus importants en quantité, tant à $\mathrm{Ny}$ Aalesund que dans nos stations. Toutefois, il faudra les exclure car le prélèvement couvre une période d'important blizzard, qui empêche de faire la part, dans la lame d'eau recueillie, entre neige tombée ( 1 et 7 octobre) et neige soufflée.

3.2 Effet de la variation d'altitude sur les quantités d'eau précipitées 


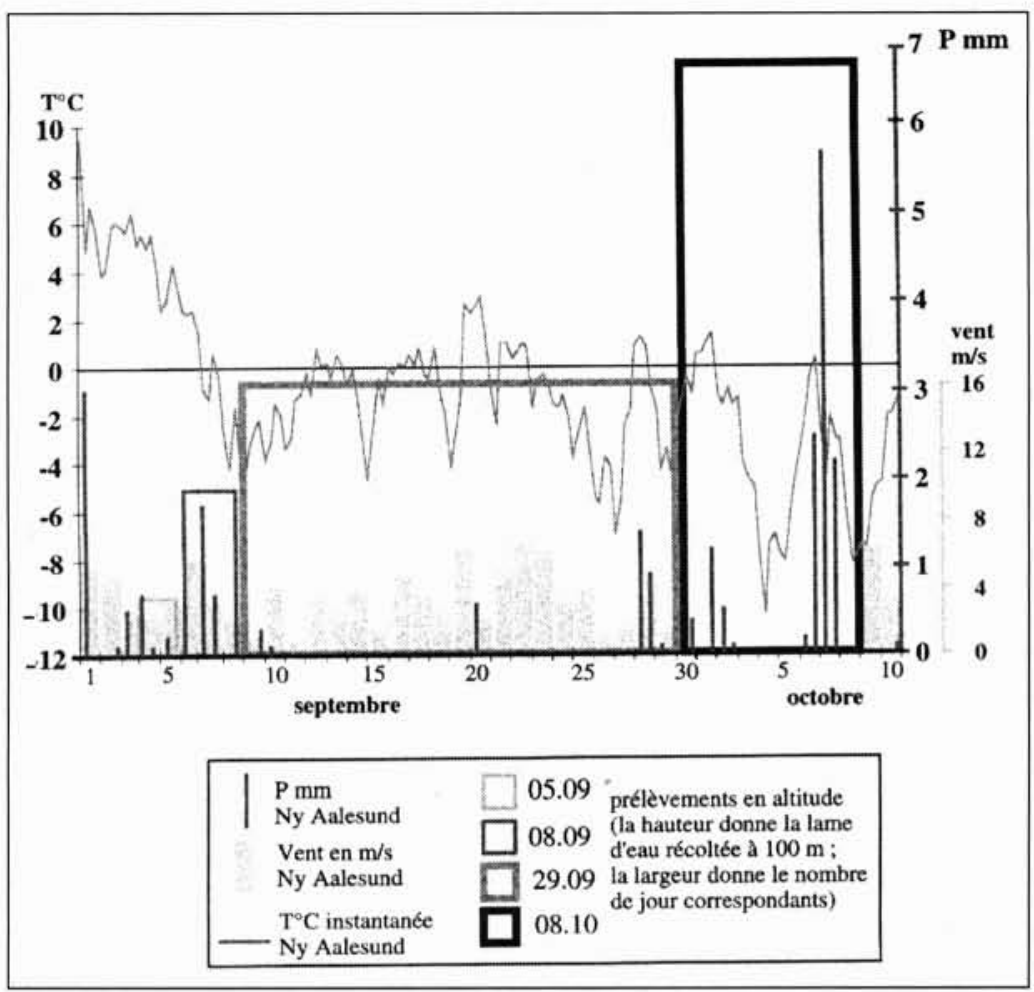

2. Températures, précipitations et vents à $\mathrm{Ny}$ Aalesund et au pied du Loven Est du 01.09.98 au 10.10.98.

\subsubsection{Gradients pluviométriques dans la littérature}

Les valeurs de gradients pluviométriques disponibles dans la littérature pour la région de la Baie du Roi sont très disparates, souvent établies à partir de deux points et sans grand détail sur la méthode employée $[8,1,9,10,2,5,4]$. Rapportés aux $400 \mathrm{~mm}$ de précipitations moyennes annuelles à $\mathrm{Ny}$ Aalesund (10 m d'altitude), ces gradients donnent différentes valeurs de hauteurs de précipitations, $P$, allant presque du simple au double à la cote $400 \mathrm{~m}\left(P_{400 \mathrm{~m}}\right.$ de 560 à $\left.800 \mathrm{~mm}\right)$, ce qui correspond à un gradient de +40 à $+100 \mathrm{~mm}$ par 100 m d'élévation (Fig. 3).

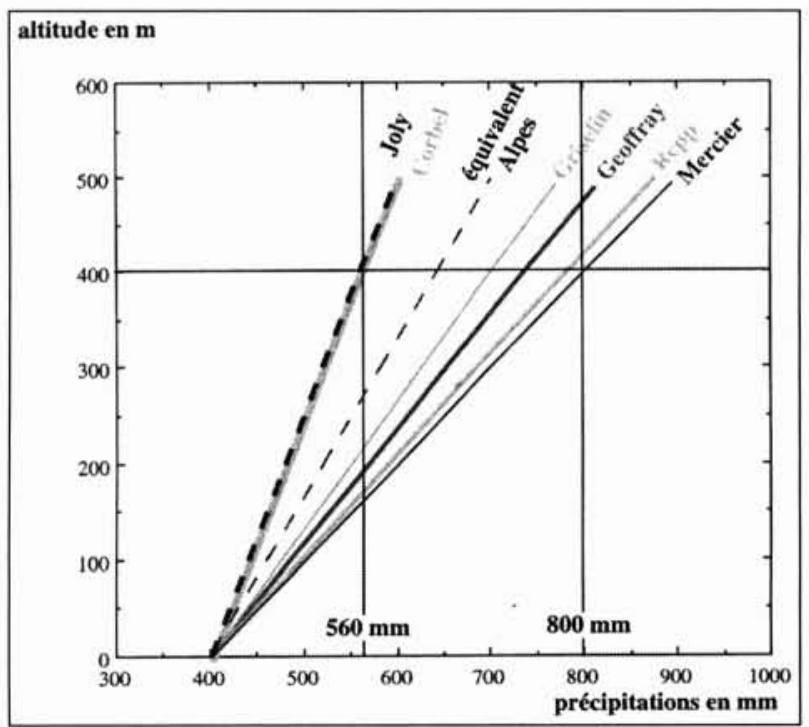

3. Gradients pluviométriques en rive sud de la baie du Roi (Spitsberg) d'après la littérature (figurés pour une hauteur précipitée annuelle de $400 \mathrm{~mm}$ à $\mathrm{Ny}$ Aalesund) et comparaison avec les Alpes.
Ces valeurs données pour la Baie du Roi sont proches des 40 à $80 \mathrm{~mm} / 100 \mathrm{~m}$ rapportées pour les Alpes occidentales [9] encore que les totaux précipités en moyenne dans les Alpes (1200 mm à $1000 \mathrm{~m}$ d'altitude) ne puissent en rien être comparés avec ceux qui caractérisent la basse montagne polaire.

\subsubsection{Gradients pluviométriques à l'échelle de l'événement}

Au cours de l'automne 1998, l'étude du gradient pluviométrique a été abordée à l'échelle de l'événement (Fig. 4 et Tab. 1). Les quatre prélèvements échantillonnés sont reportés sur la figure 4 mais le dernier, non représentatif car comportant neige tombée et neige soufflée, n'a pas été relié par des segments. Pour les mêmes raisons, la courbe de cumul indiquée sur la figure 4 ne concerne donc que les trois premiers épisodes. Même si on ne prend pas en compte les précipitations récoltées à $10 \mathrm{~m}$ d'altitude à la base française, jugée très abritée (comparativement à $\mathrm{Ny}$ Aalesund), on obtient, selon les événements, un doublement voire un triplement des valeurs entre 100 et $400 \mathrm{~m}$. Les données obtenues montrent qu'il est difficile, avec seulement quatre épisodes (dont un non représentatif), d'établir un gradient qui soit valable et extrapolable. Un gradient se dessine cependant et de façon plus nette quand les quantités précipitées sont importantes. Un optimum pluviométrique semble se situer en moyenne autour de $400 \mathrm{~m}$, ce que confirme la littérature aussi bien pour le Spitsberg [5] que pour les Alpes (supposé aux environs de $3500 \mathrm{~m}$ ), [9]. En ce qui concerne le Loven Est, cet optimum pluviométrique s'explique par la configuration du bassin : les parties plus hautes sont des cirques très froids, relativement abrités des précipitations, alors que la zone de $400 \mathrm{~m}$ est complètement ouverte et extrêmement ventée. En effet, à partir d'une certaine alti-

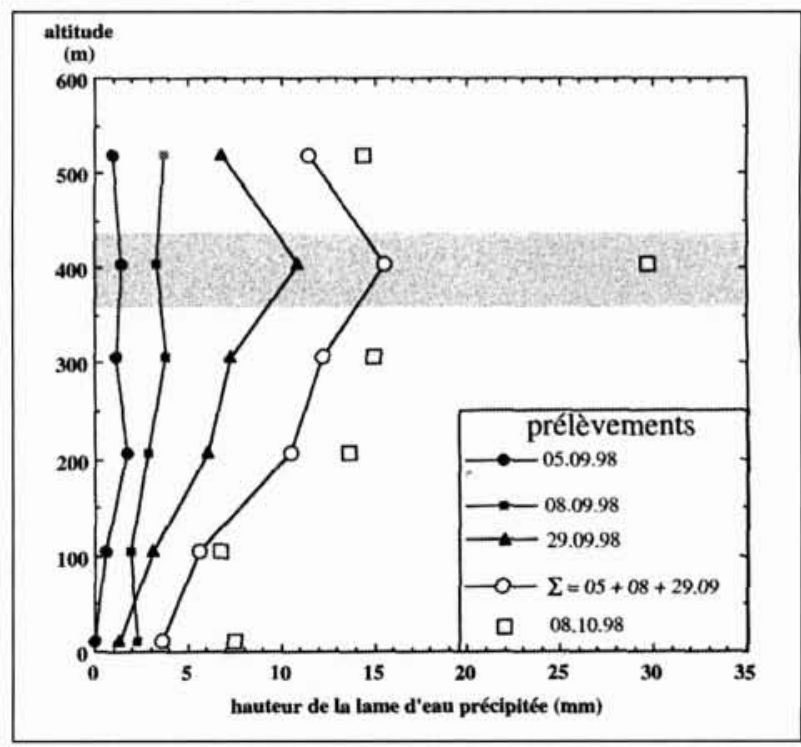

4. Variation de la lame d'eau précipitée en fonction de l'altitude (bassin du glacier Loven Est, $7^{\circ} \mathrm{N}$, Spitsberg. 


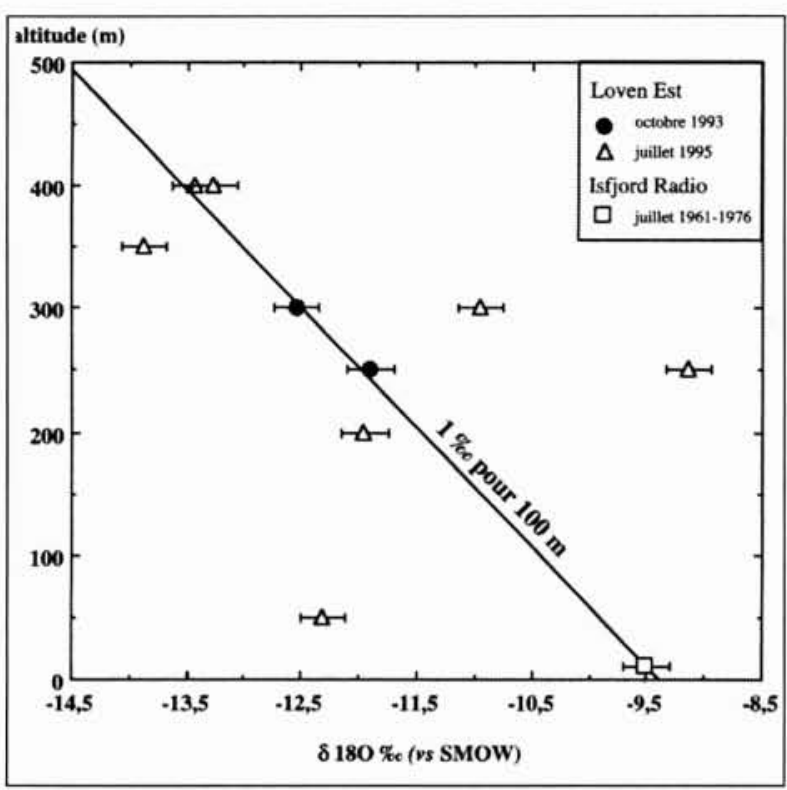

5. Gradients altitudinaux des teneurs en oxygène 18 (données de 1993 et 1995 , Loven Est, $79^{\circ} \mathrm{N}$; moyenne 1961-1976 pour Isfjord Radio, $\left.78^{\circ} \mathrm{N}\right)$.

tude, qui semble être ici $400 \mathrm{~m}$, les températures de l'air atteignent vite des valeurs qui n'autorisent plus de précipitations neigeuses. L'existence de cet optimum montre l'intérêt de faire des mesures en plusieurs points, plutôt que d'extrapoler à partir de deux points uniquement.

A l'échelle d'un travail hydrologique, seul un quadrillage de stations pluviométriques permettraient de déterminer avec précision la lame d'eau précipitée en tenant compte, en plus de l'altitude, des situations d'abri et d'exposition.

\subsection{Effet de la variation d'altitude sur la signature isotopique des précipitations}

En terme de qualité géochimique de l'eau, l'étude a porté sur les teneurs en isotopes stables de la molécule d'eau $\left({ }^{18} \mathrm{O}\right.$ et ${ }^{2} \mathrm{H}$ ), traceurs conservés de son origine à condition qu'il n'y ait pas d'évaporation ou de sublimation. En ce qui concerne la pluie, les compositions en ${ }^{18} \mathrm{O}$ et ${ }^{2} \mathrm{H}$ sont dépendantes des variations de température au moment de la précipitation, de la quantité d'eau précipitée et de l'origine de la masse d'air générant les précipitations [11]. La variation de la température et de la quantité d'eau précipitée intégrant à la fois l'effet d'altitude et de continentalité, l'oxygène 18 et le deutérium sont donc des traceurs reconnus pour montrer cette influence [12].

Dans cette étude, trois approches permettent d'aborder la caractérisation isotopique de la fonction "entrée" du système du Loven Est.

\subsubsection{Chronique de mesures isotopiques d'Isfjord-Radio}

Une chronique de données isotopiques des eaux météoriques existe pour la côte occidentale du Spitsberg. Elle a été établie à Isfjord Radio, station météorologique côtière du réseau IAEA-OMM, pour la période 1961-1976 (IAEA, 1993). Pour cette période, les valeurs annuelles varient de 7,4 à $-10,7 \%$ vs SMOW en oxygène 18 (avec une moyenne de $-9,5 \%$ et un écart-type de $1,4 \%$ ) et entre -52 et $-84 \%$ vs SMOW en deutérium (avec une moyenne de $-65 \%$ et un

\begin{tabular}{|c|c|c|c|c|}
\hline \multicolumn{5}{|c|}{ Hauteur de la lame d'eau (mm) } \\
\hline Altitude (m) & $5 / 09 / 98$ & $8 / 09 / 98$ & $29 / 09 / 98$ & $8 / 10 / 98$ \\
\hline 10 & 0,0 & 2,3 & 1,3 & 7,4 \\
\hline 105 & 0,6 & 1,9 & 3,1 & 6,7 \\
\hline 205 & 1,7 & 2,8 & 6,1 & 13,6 \\
\hline 306 & 1,1 & 3,8 & 7,3 & 14,9 \\
\hline 405 & 1,4 & 3,3 & 10,9 & 29,7 \\
\hline 520 & 0,9 & 3,7 & 6,8 & 14,4 \\
\hline Ny -A : cumul & 1 & 2,4 & 3,4 & 12,9 \\
\hline \multicolumn{5}{|c|}{$\delta^{18} \mathrm{O}(\%$ versus SMOW) } \\
\hline Altitude (m) & $5 / 09 / 98$ & $8 / 09 / 98$ & $29 / 09 / 98$ & $8 / 10 / 98$ \\
\hline 10 & & $-14,89$ & $-5,66$ & $-15,22$ \\
\hline 105 & $-5,93$ & $-14,80$ & $-6,07$ & $-13,47$ \\
\hline 205 & $-6,64$ & $-16,12$ & $-6,59$ & $-13,21$ \\
\hline 306 & $-6,71$ & $-16,43$ & $-7,12$ & $-13,36$ \\
\hline 405 & $-7,17$ & $-16,52$ & $-7,47$ & $-13,45$ \\
\hline 520 & $-6,82$ & $-16,49$ & $-8,26$ & $-12,33$ \\
\hline \multicolumn{5}{|c|}{$\delta^{2} \mathrm{H}(\%$ versus SMOW) } \\
\hline Altitude (m) & $5 / 09 / 98$ & $8 / 09 / 98$ & $29 / 09 / 98$ & $8 / 10 / 98$ \\
\hline 10 & & $-109,0$ & $-36,8$ & $-118,8$ \\
\hline 105 & $-36,2$ & $-112,9$ & $-36,6$ & $-107,7$ \\
\hline 205 & $-42,8$ & $-116,7$ & $-41,4$ & $-98,3$ \\
\hline 306 & $-45,8$ & $-122,4$ & $-41,2$ & $-104,8$ \\
\hline 405 & $-45,2$ & $-121,9$ & $-46,8$ & $-100,9$ \\
\hline 520 & $-50,1$ & $-123,5$ & $-53,2$ & $-95,0$ \\
\hline \multicolumn{5}{|c|}{ Gradients altitudinaux $(\% d 100 \mathrm{~m})$} \\
\hline & $5 / 09 / 98$ & $8 / 09 / 98$ & $29 / 09 / 98$ & $8 / 10 / 98$ \\
\hline$\Delta\left(\delta^{18} \mathrm{O}\right) / 100 \mathrm{~m}$ & $-0,22$ & $-0,38$ & $-0,50$ & $+0,40$ \\
\hline$r$ & 0,80 & 0,88 & 1,00 & 0,81 \\
\hline
\end{tabular}

Tableau 1 - Glacier Loven Est (Spitsberg) : gradients altitudinaux des hauteurs de précipitations et des teneurs en oxygène 18 et deutérium, à l'échelle de l'événement.

écart-type de $11 \%$ ). L'utilisation de ces données pour le site du Loven Est peut être validée par le fait que les deux sites, Isfjord-Radio et le versant septentrional de la presqu'île de Brögger sur lequel se situe le secteur d'étude, sont dans des conditions climatiques et géographiques relativement similaires. Toutefois, l'existence d'importantes dénivelées ( $876 \mathrm{~m}$ sur $7 \mathrm{~km}$ de longueur) sur le bassin versant nécessite d'être prise en compte : les valeurs moyennes d'IsfjordRadio peuvent-elles être extrapolables à tout le bassin ?

\subsubsection{Echantillonnage de colonnes de neige sur le bassin versant (1993 et 1995)}

La deuxième approche a consisté en un échantillonnage de colonnes de neige, à différentes altitudes à la surface du bassin versant (octobre 1993 et juillet 1995). Les mesures de teneurs en oxygène-18 ont été reportées en fonction de l'altitude en figure 5 .

Si aux données de 1993 est ajoutée la valeur moyenne des précipitations d'Isfjord Radio, un gradient isotopique de $1 \%$ o pour $100 \mathrm{~m}$ est obtenu [13]. Ce gradient est particulièrement élevé si on le compare aux valeurs habituellement rencontrées (entre 0,2 et $0,5 \%$ par $100 \mathrm{~m}$ [14]). Entre autres, un gradient altitudinal de $0,5 \%$ / $/ 100 \mathrm{~m}$ est donné pour le Mont-Blanc [15]. L'ajout, sur la figure 5, des mesures effectuées en 1995 sur un ensemble de neiges résiduelles ne semble que partiellement en accord avec les données antérieures. Si les compositions en oxygène 18 de la neige échantillonnées à $400 \mathrm{~m}$ sont dans la tendance de 1993, il ne 


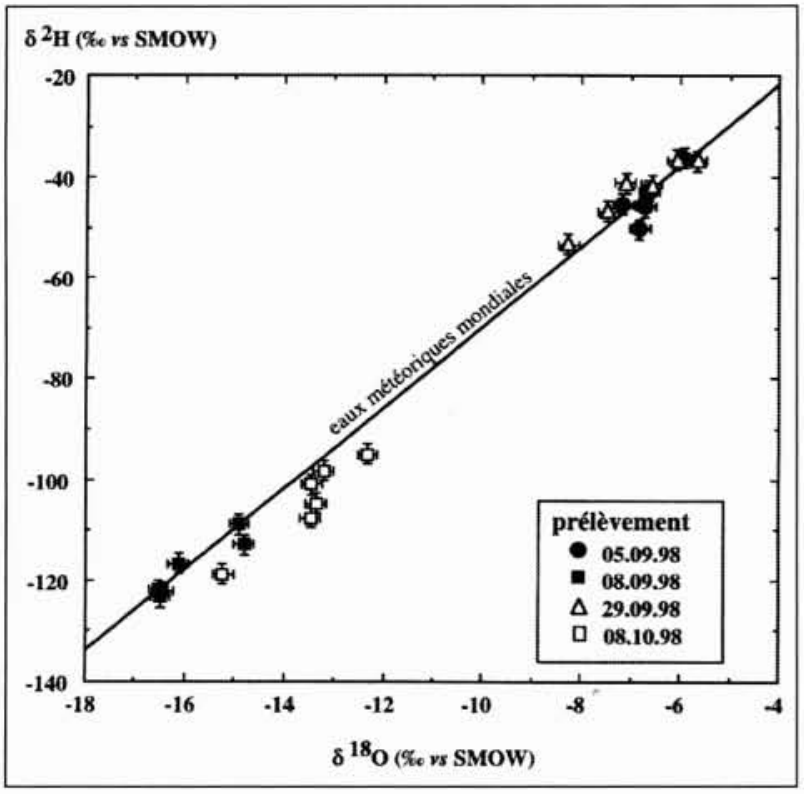

6. Teneur en deutérium et en oxygène 18 (Loven Est, $\left.79^{\circ} \mathrm{N}, 1998\right)$. La droite représentée est celle des eaux météoriques mondiales $\delta^{2} h=8 \delta^{18} \mathrm{O}+10 ;[16]$.

se dessine pas de valeur unique et encore moins de gradient unique reproductible chaque année pour l'ensemble du bassin, quand on travaille sur des colonnes de neiges résiduelles de l'hiver (ou des hivers) précédent(s). En effet, ces échantillons moyens incluent la neige soufflée, c'est-à-dire de la neige tombée à d'autres altitudes ; ils incluent aussi l'eau de fonte écoulée depuis l'amont vers l'aval et ayant imbibé la neige. Par ce processus, le signal isotopique est complètement perturbé. Cela illustre toute la complexité du système et la difficulté qui existe, en milieu périglaciaire, à caractériser un système hydrologique.

\subsubsection{Echantillonnage à l'échelle de l'événement de préci- pitations}

Une troisième approche a été expérimentée durant la période allant de septembre à octobre 1998. Ces investigations concernent la caractérisation isotopique de quatre épisodes de précipitations.

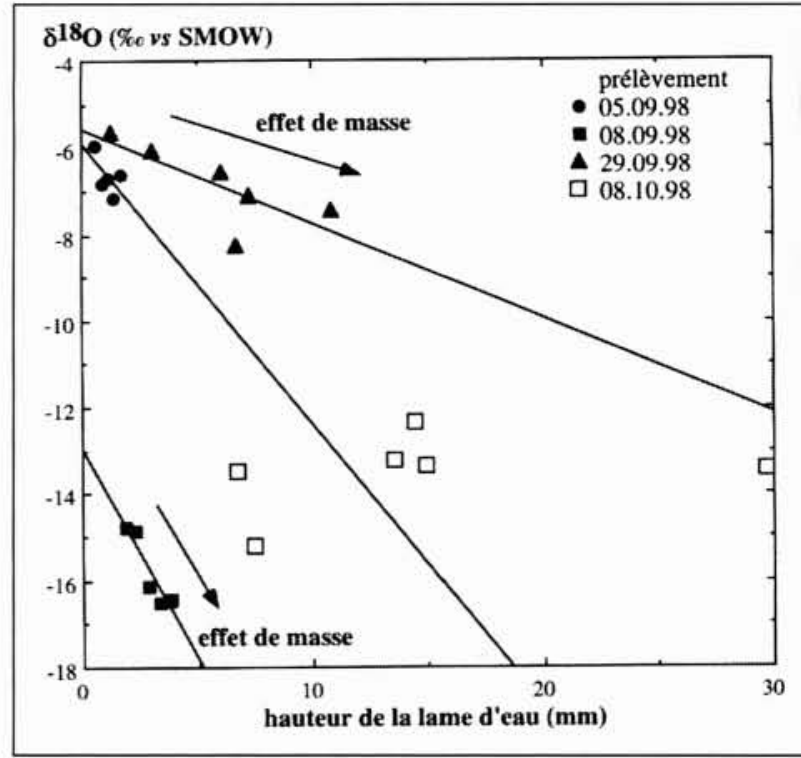

8. Teneurs en oxygène 18 en fonction de la hauteur de la lame d'eau équivalente (Loven Est, Spitsberg). Les trois droites représentent les relations linéaires existantes entre $\delta^{18} \mathrm{O}$ et la hauteur des précipitations pour les épisodes du 04.09 (cercles pleins), 07.09 (carrés pleins) et du 28.09 .98 (triangle pleins).

Reportés dans un diagramme $\delta^{2} \mathrm{H}$ en fonction de $\delta^{18} \mathrm{O}$ (Fig. 6), les points analytiques représentant les différents épisodes se répartissent le long de la droite des eaux météoriques mondiales [16]. Cette disposition indique que les neiges n'ont pas subi de sublimation lors de leur chute dans le profil atmosphérique ni lors de leur séjour dans les pluviomètres. Ainsi, des pertes en eaux précipitées ou une modification de la composition isotopique des neiges échantillonnées par ce processus, si elles existent, ne sont pas significatives.

Les données obtenues montrent que les teneurs en ${ }^{18} \mathrm{O}$ et ${ }^{2} \mathrm{H}$ des neiges prélevées à différentes altitudes varient largement d'un épisode à l'autre tant dans le temps que dans l'espace (Fig. 7 et Tab. 1). Sur seulement quatre épisodes pluvio-neigeux, ces variations sont considérables, puisque égales à $9 \%$ en oxygène $18\left(\delta^{18} \mathrm{O}\right.$ de -6 et $-15 \%$ et à $84 \%$ en deutérium $\left(\delta^{2} \mathrm{H}\right.$ de -36 et $-120 \%$ o $)$. L'impact des variations de la température de l'air semble négligeable à l'échelle de la période d'observation. Pour une même altitude, ces variations se sont révélées indépendantes de la température, de la hauteur de précipitations ou de l'origine des masses d'air (Fig. 2 et 7, Tab. 1). Une explication serait à rechercher dans la température de la base des nuages et/ou dans l'historique des masses d'air générant les précipitations.

En excluant le prélèvement du 08.10 (neige soufflée), on observe un appauvrissement des précipitations avec l'altitude en ${ }^{18} \mathrm{O}$ selon des gradients significatifs et compris entre 0,2 et $0,5 \%$ par $100 \mathrm{~m}$ (Fig. 7). Echantillon le plus fiable par les quantités d'eaux précipitées et présentant un coefficient de corrélation de 1,00 entre $\mathrm{d}^{18} \mathrm{O}$ et l'altitude, le prélèvement du 29.09 est
7. Gradients altitudinaux des teneurs en oxygène 18 pour différents épisodes de précipitations (bassin du glacier Loven Est, Spitsberg). 
caractérisé par le gradient altitudinal le plus élevé de la série $(0,5 \%$ / $100 \mathrm{~m})$, du même ordre, d'ailleurs, que celui établi pour le Mont-Blanc, [15].

Une dépendance entre la teneur en ${ }^{18} \mathrm{O}$ et la hauteur d'eau précipitée existe pour les trois épisodes retenus (Fig. 8). L'appauvrissement isotopique avec l'altitude semble lié à la fois à un effet de masse et à un effet de température (3 à $5^{\circ} \mathrm{C}$ d'écart entre $10 \mathrm{~m}$ et $300 \mathrm{~m}$ ).

\section{IV $\square$ CONCLUSION}

L'effet de l'altitude sur le gradient pluviométrique et isotopique des précipitations est indéniable. Cependant, la fonction "entrée" du système hydrologique étudié est loin d'être homogène et pose, à la fois qualitativement et quantitativement, le problème de la représentativité de l'échantillonnage. L'idéal serait de coupler un travail à l'échelle des événements avec le prélèvement de colonnes de neige en fin de période d'accummulation (mai-juin) avant même que la fonte ne commence. Mais quelle serait la représentivité de l'échantillonnage d'un hiver pour pouvoir comparer la fonction "entrée" avec les écoulements pérennes observés sous le Loven Est et dont les temps de séjour sont de l'ordre de quelques années ?

En milieu polaire océanique, même sur des bassins versants de petites dimensions, les investigations réalisées à différentes échelles montrent qu'il faudrait envisager un véritable quadrillage du bassin pour déterminer de la façon la plus exacte possible la fonction "entrée". Seule une telle démarche permettrait la prise en compte de la variabilité spatiale des précipitations, dépendantes, en plus de l'altitude, des situations d'abri, de l'exposition et des effets de mélange des eaux lié au vent et à la fonte. La preuve est faite que, même sur de très petites unités hydrologiques, l'échantillonnage des précipitations au niveau de la mer en zone polaire, s'il est commode, n'est en tout cas pas extrapolable à l'ensemble des bassins.

\section{Remerciements}

Sandra Van Exter pour avoir fait les mesures isotopiques (Laboratoire d'hydrologie et de Géochimie isotopique, Université de Paris-Sud). Philippe Barthez, Dominique Laffly, Denis Mercier et Christiane Salvador pour leur aide sur le terrain.

\section{Références}

[1] GEOFFRAY H., 1968 : Etude du bilan hydrologique et de l'érosion sur un bassin partiellement englacé du Spitsberg, Baie du Roi, $79^{\circ} \mathrm{N}$, Thèse de $3^{\mathrm{e}}$ cycle, Brest, $68 \mathrm{p}$.

[2] GRISELIN M., 1982 : Les écoulements liquides et solides sur les marges polaires : exemple du bassin Loven Est, côte nord-ouest du Spitsberg, thèse de $3^{\mathrm{e}}$ cycle, volume III des travaux du laboratoire de géographie physique de Nancy II, $500 \mathrm{p}$.

[3] VIVIAN H., 1964 : "Premières observations sur le régime estival des torrents glaciaires du Spitsberg $\left.\left(79^{\circ} \mathrm{N}\right)\right)^{*}$, Norois, $\mathrm{n}^{\circ} 43$, pp. 283-307.

[4] MERCIER D., 1998 : Le ruissellement au Spitsberg : l'impact d'un processus azonal sur les paysages d'un milieu polaire, presqu'île de Brøgger $\left(79^{\circ} \mathrm{N}\right)$, thèse, université de Clermont-Ferrand, $531 \mathrm{p}$.

[5] JOLY D., 1994 : Ambiances climatiques instantanées au Spitsberg, pour une approche méthodique par niveaux d'échelle, thèse d'Etat, EHESS, annales littéraires de l'université de Franche-Comté, Paris, $n^{\circ}$ 529, 404 p.

[6] Mc CREA J., 1950. "On the Isotope Chemistry of Carbonates and Palaeotemperature Scales" J. of Chem. Phys. 18 : pp. 849-857.

[7] COLEMAN M.L., SHERPHERDT.J., DURHAM J.J., ROUSE J.E. and MOORE G.R., 1982. "Reduction of Water with Zinc for Hydrogen Isotope Analysis", Anal. Chem. 54 ; pp. 993-995.

[8] CORBEL J., 1966 : "Recherches sur les climats du Spitsberg et leurs variations récentes", in Spitsberg 1964, Lyon, pp. 41-54.

[9] GAUDET F., 1975 : Les cours d'eau alpins de régime glaciaire, Thèse, Brest, Lille III, 417 p.

[10] REPP K., 1979 : Breerosjon, glasio-hydrologi og materialtransport i et høyarktisk miljo - Brøggerbreene, Vestspitsbergen, universite i Oslo, mémoire de maîtrise, 138 p.

[11] YURTSEVER Y. et GAT J.R., 1981 : “Atmospheric Waters". In Deuterium and Oxygen-18 in the Water Cycle, Technical reports series 210 , A.I.E.A., Vienne : pp. 103-142.

[12] FONTES J.Ch., 1976 : Isotopes du milieu et cycles des eaux naturelles : quelques aspects, Thèse de doctorat ès Sciences, Univ. Paris VI, 208 p.

[13] GRISELIN M., MARLIN Ch., MOREAU L. et DEVER L., 1995 : "Hydrologie et géochimie du glacier Loven Est (Spitsberg)", Actes du $3^{*}$ symposium Cavités glaciaires et cryokarst en régions polaires et de hautes montagnes, article bilingue français-anglais, (pp. 61-76).

[14] BLAVOUX B., 1978 : Etude du cycle de l'eau de l'oxygène 18 et du tritium. Possibilités et limites de la méthode des isotopes du milieu en hydrologie dans la zone tempérée. Thèse de Doctorat ès Sciences, Univ. Paris VI, 333 p.

[15] MOSER H. et STRICHLER W, 1970 : "Deuterium Measurements on Snow Samples from the Alps. Isotope Hydrology ", Symp. Proceedings, I.A.E.A., Vienne : 43-57.

[16] CRAIG. H., 1961. "Isotopic Variations in Meteoric Waters", Science, 133, pp. 1833-1834. 